\title{
Analytical method development for quantitation of adenosine and cordycepin in cordyceps and related products
}

\section{Jinda Wangboonskul ${ }^{1 *}$, \\ Surattana Amnuoypol ${ }^{1}$, \\ Paradi Sangvatanakul ${ }^{2}$}

${ }^{1}$ Division of Pharmaceutical Sciences, Faculty of Pharmacy, Thammasat University, Pathumthani, Thailand

${ }^{2}$ Division of Traditional Chinese Medicine, Chulabhorn International College of Medicine, Thammasat University, Pathumthani, Thailand

*Corresponding author: Jinda Wangboonskul jinda_wa@tu.ac.th

\section{KEYWORDS:}

Cordyceps sinensis; Cordyceps capsules; Adenosine; Cordycepin https://www.pharmacy.mahidol.ac.th/journal/ (C) Faculty of Pharmacy, Mahidol University (Thailand) 2020

\begin{abstract}
Cordyceps products which were produced from biotechnology processes, were widely advertised as a health promotor. They were aimed to replace the natural Cordyceps sinensis $(C$. Sinensis) with much cheaper price. The quality of the products may be related to the level of main active compounds, adenosine and cordycepin. The objectives of this study is to develop an analytical method for quantifying the level of adenosine and cordycepin. The analytical method was undertaken by gradient elution with methanol and $0.1 \%$ Diisopropylamine in water adjusted to $\mathrm{pH} 6.0 \pm 0.1$ as mobile phases, on an Hypersil ODS column $(4.0 \times 250 \mathrm{~mm}, 5 \mu \mathrm{m})$, at flow rate $1.0 \mathrm{~mL} / \mathrm{min}$ and detected with a diode array detector. The pure compounds of adenosine and cordycepin were used as standard. The developed analytical method was proved specific, reliable and sensitive to adenosine and cordycepin contained in natural $C$. sinensis and in cordyceps capsules. Calibrations were linear over the range $2.56-392 \mu \mathrm{g} / \mathrm{mL}$ for adenosine, and $17.2-488 \mu \mathrm{g} / \mathrm{mL}$ for cordycepin with the correlation coefficient $\left(r^{2}\right)$ of 1 and 0.9999 , respectively. The percentage recovery of adenosine and cordycepin were 99.47-101.35 and 98.80-101.49, respectively. The RSD of both repeatability and intermediate precision were below $2 \%$. The limits of quantification for adenosine and cordycepin were 1.13 and 2.78 $\mu \mathrm{g} / \mathrm{mL}$, respectively. The method was applied to determine the levels of adenosine and cordycepin in cordyceps capsules. The amount of adenosine and cordycepin were vary among samples: adenosine was from less than LOQ to $1.613 \mathrm{mg} / \mathrm{capsule}$ and cordycepin was from less than LOQ to $3.435 \mathrm{mg} /$ capsule.
\end{abstract}

\section{INTRODUCTION}

Natural C. sinensis or Chinese caterpillar fungus have been recorded as a health promotor for more than two centuries in China. It consists of 2 parts: worm (Hepialus armoricanus Oberth ̈̈r) and mycelium of fungus (Ophiocordyceps sinensis former name is Cordyceps sinensis) $)^{1}$. It is rare and expensive, so that part of fungus has been cultivated by biotechnology processes; aims for replacing the natural Cordyceps. The 
products are in various forms such as capsules, tea, coffee, etc. The active constituents of $C$. sinensis may be divided to six groups; nucleosides, polysaccharides, sterols, proteins, vitamins and minerals ${ }^{1}$. All were claimed for health benefit ${ }^{2}$. Adenosine was used as the biomarker for $C$. sinensis in The Pharmacopoeia of the People's Republic of China, $2005^{3}$. The level of active compounds, adenosine and cordycepin, obtained from cultivation was proved not inferior to the natural C. Sinensis ${ }^{2}$. There are three fermented preparation of $C$. Sinensis in The Pharmacopoeia of the People's Republic of China, 20154, Bailing Jiaonang, Jinshuibao Jiaonang and Jinshuibao Pian. Adenosine and cordycepin have similar basic chemical structure as shown in Figure 1, both are nucleosides. Adenosine was approved by the U.S.FDA for the treatment of supraventricular tachycardia in $1990^{5}$. Cordycepin was also suggested the same indication as adenosine ${ }^{6}$. Adenosine and cordycepin were recommended to use as biomarkers for quality control for natural C. sinensis and cordyceps products ${ }^{2}$.

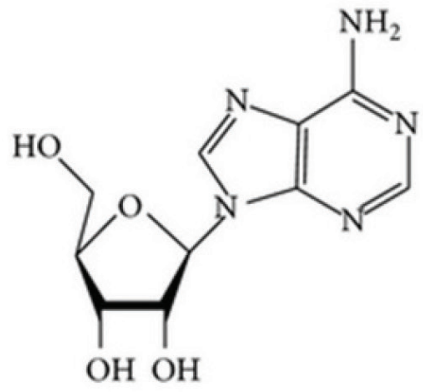

Adenosine

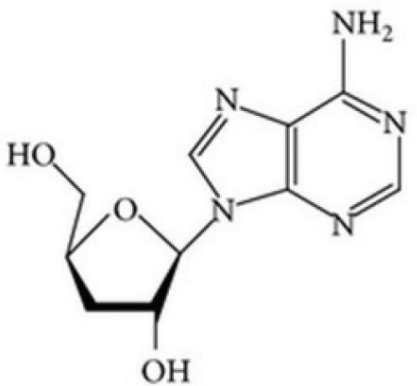

Cordycepin

Figure 1. The chemical structures of adenosine and cordycepin

Analysis method for quality control of Cordyceps has been developed, from the simple ways but lack of specificity e.g. Redox titration, Colorimetry, Thin layer chromatography. More selective methods using high performance equipment e.g. Gas chromatography, High performance liquid chromatography (HPLC) and Capillary electrophoresis have been reported ${ }^{2,7}$. HPLC maybe the most important tool for quantitative analysis for the active constituents in Cordyceps.

\section{MATERIALS AND METHODS}

\subsection{Materials}

The purity of standards, adenosine and cordycepin, were over $98 \%$. Both were purchased from Sigma-Aldrich, Saint Louis, USA. HPLCgrade methanol was purchased from Merck Co., (Merck, Darmstadt, Germany). Diisopropylamine was obtained from Fluka (Chemika, UK. Deionized water was purified by MilliQ system (Millipore, Bedford, MA, USA). Other chemicals were of reagent grade. $0.45 \mu \mathrm{m}$ Nylon syringe filter was purchased from Agela Technologies, China.

\subsection{Methods}

\subsubsection{Chromatographic condition}

High-performance liquid chromatography analysis was carried out on a Shimadzu system, equipped with a low pressure gradient pumps 4 channels (LC-20AD), vacuum degasser (DGU$20 \mathrm{~A}_{5 \mathrm{R}}$ ), autosampler (SIL 20AC $\mathrm{HT}_{\mathrm{T}}$ ), column oven (CTO 20AC), a diode array detector (SPD-M20A) and a CBM-20A System Controller. All data acquired were proceeded by LabSolutions software (Shimadzu Japan).

Chromatographic separation was performed at $30^{\circ} \mathrm{C}$ using an Hypersil ODS column $(4.0 \times 250$ $\mathrm{mm}, 5 \mu \mathrm{m}$ ) with a $\mathrm{C}_{18}$ guard column supplied by Waters. The mobile phase consisted of the mixture of $0.1 \%$ Diisopropylamine in water adjusted to $\mathrm{pH} 6.0 \pm 0.1$ with phosphoric acid (A) and methanol (B). The initial composition was $98 \% \mathrm{~A}$ and $2 \% \mathrm{~B}$. The gradient program was as follows; $03 \mathrm{~min}, 98 \%$ A; 3-10 min, $90 \%$ A; $10-30 \min , 78 \%$ A; 30-45 $\min , 0 \% \mathrm{~A} ; 4560 \mathrm{~min}, 98 \% \mathrm{~A}$. The stop time and post time were set at 45 and $3 \mathrm{~min}$, respectively. The mobile phase was delivered to the column at 
a flow rate of $1.0 \mathrm{~mL} / \mathrm{min}$ and the eluates were detected at $254 \mathrm{~nm}$. Ten microliters of sample were injected into the HPLC system.

\subsubsection{Method validation}

Specificity of the method for adenosine and cordycepin was carried out by assessing the peak identity using \%RSD $<2$ for the retention time and the purity index. The purity index is a measure of the peak's relative purity ${ }^{8}$. The peak purity index of adenosine and cordycepin in samples was demonstrated the selectivity of the method. Due to the formula of most of the cordyceps capsules were consisted of others health promotors, the criteria of purity index value was then set at $>0.9$ in this study. The system linearity was performed to ensure the range of the calibration curves covered high variation levels in samples, fourteen concentrations were used. Pure compounds of adenosine and cordycepin were prepared in water with the range of $19.6-392.0 \mu \mathrm{g} / \mathrm{mL}$ and $18.3-488.0 \mu \mathrm{g} / \mathrm{mL}$, respectively, before $10 \mu \mathrm{L}$ injecting into an HPLC. The calibration curves were plotted and coefficient of determination was calculated. The limit of detection(LOD) and limit of quantification (LOQ) were determined by calculating from five calibration lines using equations $3.3 \sigma / \mathrm{S}$ and $10 \sigma / \mathrm{S}$, respectively. When $\sigma$ is the standard deviation of $\mathrm{y}$-intercepts of regression lines and $\mathrm{S}$ is the slope of the calibration curve ${ }^{9}$. The repeatability was performed by four concentrations each repeated analysis for 3 injections on a single day. The intermediate precision was carried out on 3 different days. Variations were expressed by the percentage relative standard deviations (\%RSD). For accuracy, four concentrations of the known value of adenosine and cordycepin, were prepared and injected in triplicate. The found concentration of each analyte was analyzed within the same day. The method was then applied to determine amount of adenosine and cordycepin contained in samples.

\subsection{Cordyceps samples preparation}

\subsubsection{Natural C. sinensis preparation}

Weigh 5 natural C. sinensis, ground to powder. Accurately weighed 0.1 gram into a test tube with screw caps, soaked with $2 \mathrm{~mL} 50^{\circ} \mathrm{C}$ water for 1 hour, vortex and then filtered through a 0.45 $\mu \mathrm{m}$ Nylon syringe filter before $10 \mu \mathrm{L}$ injecting into an HPLC.

\subsubsection{Cordyceps capsules preparation}

28 samples of cordyceps capsules were purchased via the advertised media of TV, cable $\mathrm{TV}$, website and trade fair. One capsule of each sample was used, shell was removed and placed into separate test tubes with screw caps. All samples were soaked in $50^{\circ} \mathrm{C}$ water for 1 hour, volume of water added depended on the labeled of each product to give approximately $50 \mathrm{mg} / \mathrm{mL}$ of Cordyceps. Vortex and then filtered through a $0.45 \mu \mathrm{m}$ Nylon syringe filter before $10 \mu \mathrm{L}$ injecting into an HPLC.

\section{RESULTS}

Method validation

Specificity

The method shows the ability to measure accurately and specific to adenosine and cordycepin in the presence of components that may be present in the sample matrix. There was no interfering peak at the same retention time of adenosine and cordycepin. The retention time of adenosine and cordycepin $(n=176)$ over the analysis time of 8 days were $17.91 \pm 0.06 \mathrm{~min}$ and $20.40 \pm 0.08 \mathrm{~min}$, respectively. These showed the peak identity with the $\%$ RSD less than 2 . The selectivity of the method was also presented by peak purity index as shown in Figure 2, only 1 out of 28 samples for each of adenosine and cordycepin gave value of less than 0.9, however, was greater than 0.8.

The correlation coefficients $\left(r^{2}\right)$ of adenosine and cordycepin were 1 and 0.9999, respectively. The linear regression equations of adenosine and cordycepin were $\mathrm{y}=29636 \mathrm{x}$ 8609.6 and $y=27529 x-21805$, respectively. This indicated the acceptable correlations between concentrations of adenosine and cordycepin and their peak areas within the test ranges of 2.56$392 \mu \mathrm{g} / \mathrm{mL}$ and $17.2-488 \mu \mathrm{g} / \mathrm{mL}$, respectively, as shown in Figure 3. 

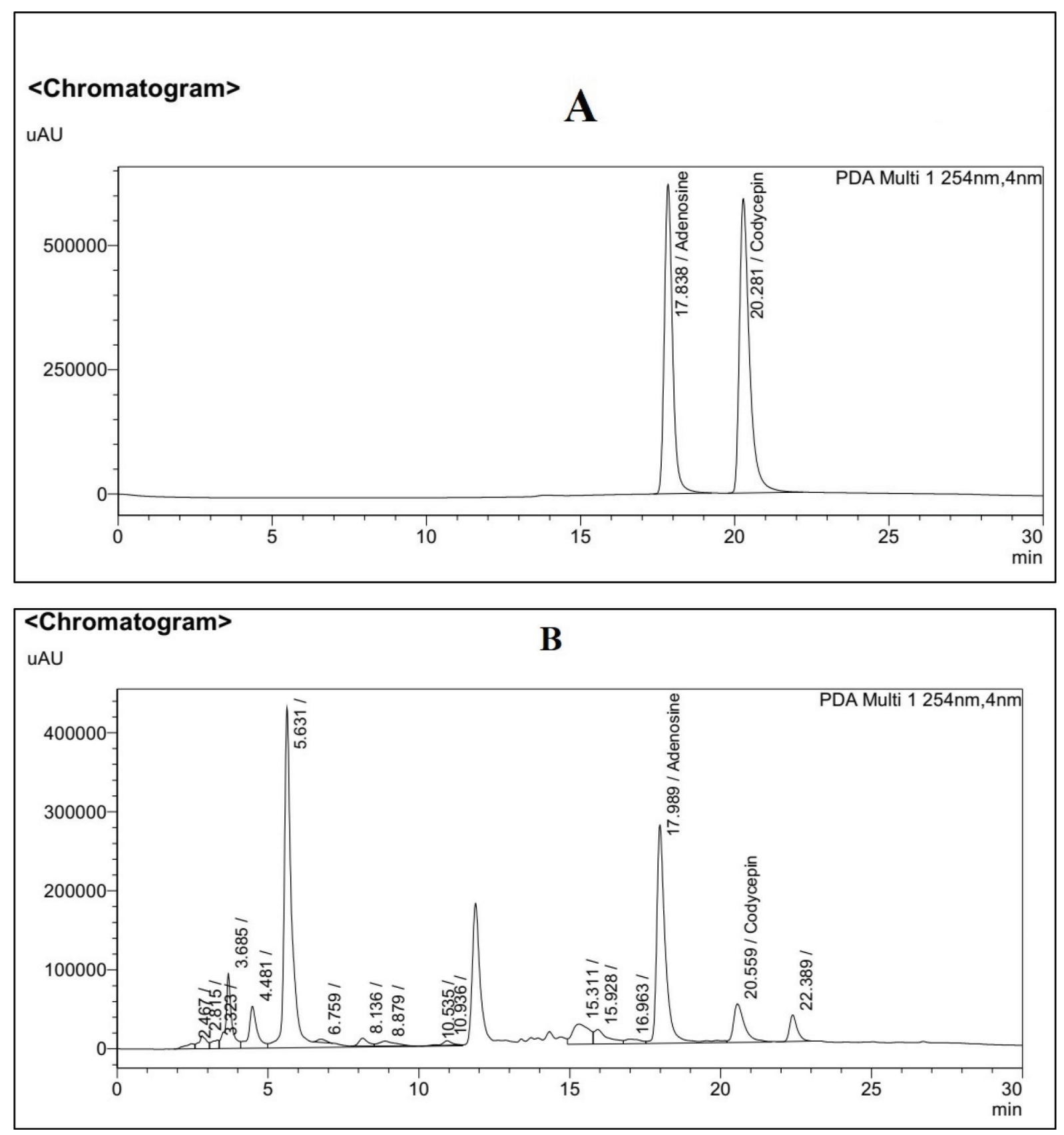

Figure 2. Chromatograms were from standard compounds of adenosine and cordycepin (A) and from one cordyceps sample (B), respectively 


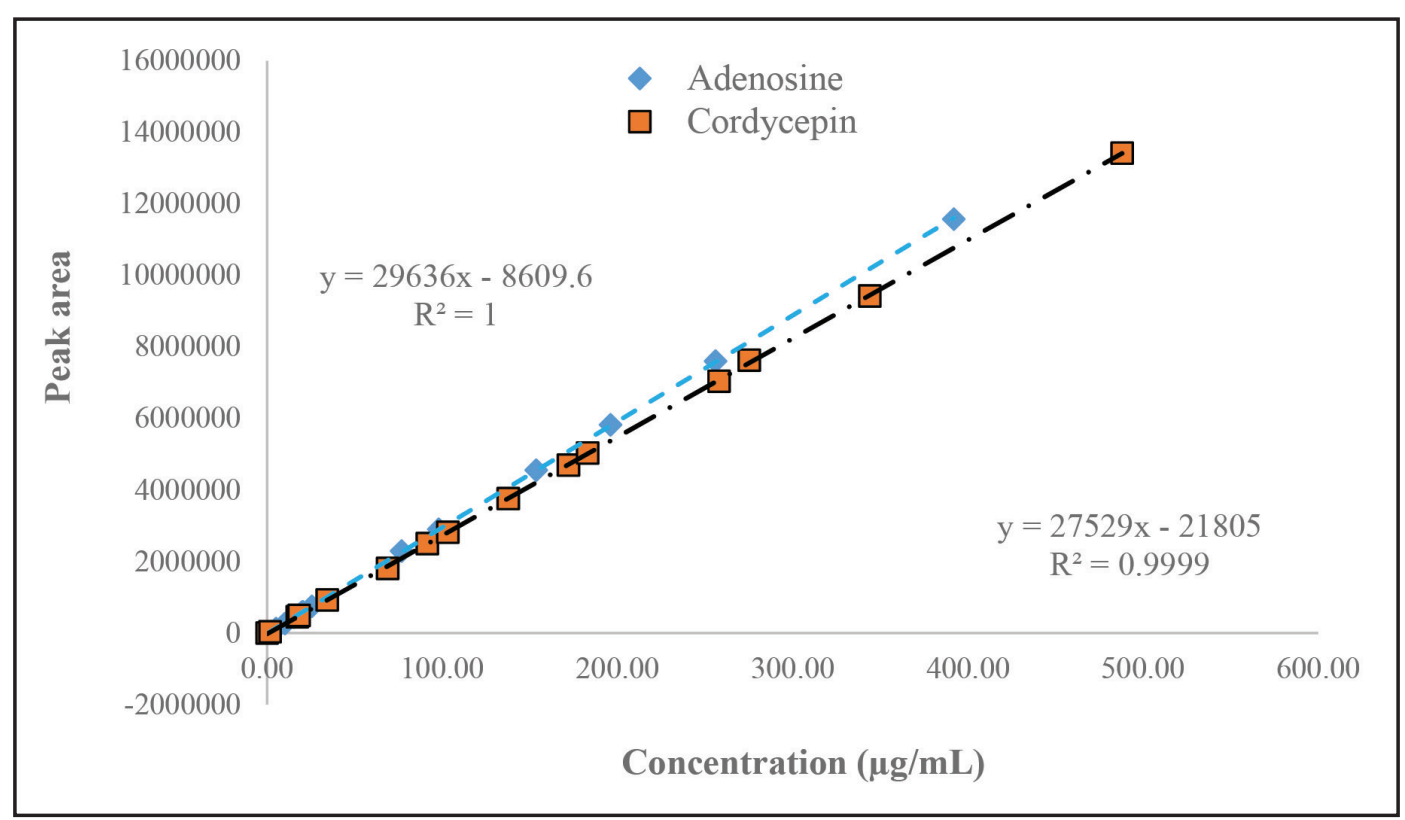

Figure 3. The calibration curves of adenosine and cordycepin, concentrations $(\mu \mathrm{g} / \mathrm{mL})$ were plotted against peak areas

Accuracy and precision

Good accuracy, repeatability and intermediate precision (3 days) of the method for adenosine determination was shown by the overall recovery of $99.47101 .35 \%$, with the $\%$ RSD ranging from
0.02 to 0.30 as illustrated in Table 1 . Table 2 shows the accurate and precise of the method for determination of cordycepin, the percentage recovery was from $98.80-101.49 \%$ with the $\%$ RSD of 0.02 to 0.45 .

Table 1. Accuracy and precision of adenosine determination obtained from the method in this study

\begin{tabular}{|c|c|c|c|c|}
\hline Adenosine & Amount added $(\boldsymbol{\mu g} / \mathbf{m L})$ & Amount found $(\boldsymbol{\mu g} / \mathbf{m L})$ & \%Recovery & \%RSD \\
\hline Repeatability $\mathbf{n}=\mathbf{3}$ & & & & \\
\hline & 392.0 & $389.93 \pm 1.16$ & $99.47 \pm 0.30$ & 0.30 \\
\hline & 196.0 & $196.76 \pm 0.07$ & $100.39 \pm 0.04$ & 0.04 \\
\hline & 98.0 & $98.33 \pm 0.07$ & $100.34 \pm 0.07$ & 0.07 \\
\hline & 19.6 & $19.87 \pm 0.01$ & $101.35 \pm 0.05$ & 0.05 \\
\hline Intermediate precision $\mathbf{n = 3}$ & & & & \\
\hline $\mathbf{1}^{\text {st }}$ day & 392.0 & $389.93 \pm 1.16$ & $99.47 \pm 0.30$ & 0.30 \\
\hline & 196.0 & $196.76 \pm 0.07$ & $100.39 \pm 0.04$ & 0.04 \\
\hline & 98.0 & $98.33 \pm 0.07$ & $100.34 \pm 0.07$ & 0.07 \\
\hline & 19.6 & $19.87 \pm 0.01$ & $101.35 \pm 0.05$ & 0.05 \\
\hline $\mathbf{2}^{\text {nd }}$ day & 392.0 & $391.10 \pm 0.93$ & $99.77 \pm 0.24$ & 0.24 \\
\hline & 196.0 & $196.74 \pm 0.12$ & $100.38 \pm 0.06$ & 0.06 \\
\hline & 98.0 & $98.38 \pm 0.02$ & $100.38 \pm 0.02$ & 0.02 \\
\hline & 19.6 & $19.95 \pm 0.00$ & $101.79 \pm 0.02$ & 0.02 \\
\hline $\mathbf{3}^{\text {rd }}$ day & 392.0 & $391.50 \pm 0.13$ & $99.87 \pm 0.03$ & 0.03 \\
\hline & 196.0 & $196.62 \pm 0.12$ & $100.31 \pm 0.06$ & 0.06 \\
\hline & 98.0 & $98.29 \pm 0.16$ & $100.29 \pm 0.16$ & 0.16 \\
\hline & 19.6 & $19.85 \pm 0.02$ & $101.29 \pm 0.10$ & 0.10 \\
\hline
\end{tabular}


Table 2. Accuracy and precision of cordycepin determination obtained from the method in this study

\begin{tabular}{|c|c|c|c|c|}
\hline Cordycepin & Amount added $(\boldsymbol{\mu g} / \mathbf{m L})$ & Amount found $(\boldsymbol{\mu g} / \mathbf{m L})$ & \%Recovery & \%RSD \\
\hline Repeatability $\mathbf{n}=\mathbf{3}$ & & & & \\
\hline & 488.0 & $487.97 \pm 0.81$ & $99.99 \pm 0.17$ & 0.17 \\
\hline & 183.0 & $183.55 \pm 0.11$ & $100.30 \pm 0.06$ & 0.06 \\
\hline & 91.5 & $91.68 \pm 0.02$ & $100.20 \pm 0.02$ & 0.02 \\
\hline & 18.3 & $18.57 \pm 0.04$ & $101.49 \pm 0.22$ & 0.22 \\
\hline Intermediate precision $\mathbf{n}=\mathbf{3}^{\text {st }}$ day & & & & \\
\hline & 488.0 & $487.97 \pm 0.81$ & $99.99 \pm 0.17$ & 0.17 \\
\hline & 183.0 & $183.55 \pm 0.11$ & $100.30 \pm 0.06$ & 0.06 \\
\hline & 91.5 & $91.68 \pm 0.02$ & $100.20 \pm 0.02$ & 0.02 \\
\hline $\mathbf{2}^{\text {nd }}$ day & 18.3 & $18.57 \pm 0.04$ & $101.49 \pm 0.22$ & 0.22 \\
\hline & 488.0 & $489.93 \pm 1.26$ & $100.40 \pm 0.26$ & 0.26 \\
\hline & 183.0 & $183.31 \pm 0.20$ & $100.17 \pm 0.11$ & 0.11 \\
\hline & 91.5 & $91.02 \pm 0.12$ & $99.48 \pm 0.14$ & 0.14 \\
\hline $\mathbf{3}^{\text {rd }}$ day & 18.3 & $18.36 \pm 0.08$ & $100.30 \pm 0.46$ & 0.45 \\
\hline & 488.0 & $490.33 \pm 0.67$ & $100.48 \pm 0.14$ & 0.14 \\
\hline & 183.0 & $183.20 \pm 0.20$ & $100.11 \pm 0.11$ & 0.11 \\
\hline & 91.5 & $90.90 \pm 0.02$ & $99.35 \pm 0.02$ & 0.02 \\
\hline & 18.3 & $18.08 \pm 0.02$ & $98.80 \pm 0.11$ & 0.11 \\
\hline
\end{tabular}

The limits of detection (LOD) and limit of quantitation (LOQ)

The LOD and LOQ was obtained by calculating from five calibration curves as shown in Table 3. The LOD of adenosine and cordycepin was $0.37 \mu \mathrm{g} / \mathrm{mL}$ and $0.92 \mu \mathrm{g} / \mathrm{mL}$, respectively. The LOQ of adenosine and cordycepin was 1.13 $\mu \mathrm{g} / \mathrm{mL}$ and $2.78 \mu \mathrm{g} / \mathrm{mL}$, respectively.

Table 3. LOD and LOQ were calculated by using five calibration curves obtained from the method in this study

\begin{tabular}{|c|c|c|c|c|c|c|}
\hline \multirow{2}{*}{ Calibration curves } & \multicolumn{3}{|c|}{ Adenosine } & \multicolumn{3}{|c|}{ Cordycepin } \\
\hline & $r^{2}$ & slope & intercept & $r^{2}$ & slope & intercept \\
\hline $1^{\text {st }}$ calibration curve & 0.9999 & 29433 & -14002 & 1 & 27482 & -15296 \\
\hline $2^{\text {nd }}$ calibration curve & 0.9999 & 29428 & -16240 & 1 & 27303 & -20193 \\
\hline $3^{\text {rd }}$ calibration curve & 0.9999 & 29412 & -13181 & 1 & 27328 & -20960 \\
\hline $4^{\text {th }}$ calibration curve & 1 & 29636 & -8609.6 & 0.9999 & 27529 & -21805 \\
\hline $5^{\text {th }}$ calibration curve & 0.9998 & 29602 & -8902.6 & 0.9999 & 27460 & -3503.8 \\
\hline Mean & 0.9999 & 29502(S) & -12187 & 1.0000 & $27420(\mathrm{~S})$ & -16351 \\
\hline Standard deviation & 0.0001 & 107.6 & $3328\left(0^{\circ}\right)$ & 0.0001 & 99.3 & $7614\left(0^{\prime}\right)$ \\
\hline $\mathrm{LOD}=\mathbf{3 . 3 0} / \mathrm{S}$ & \multicolumn{3}{|c|}{$=0.37 \mu \mathrm{g} / \mathrm{mL}$} & \multicolumn{3}{|c|}{$=0.92 \mu \mathrm{g} / \mathrm{mL}$} \\
\hline$L O Q=100 / S$ & \multicolumn{3}{|c|}{$=1.13 \mu \mathrm{g} / \mathrm{mL}$} & \multicolumn{3}{|c|}{$=2.78 \mu \mathrm{g} / \mathrm{mL}$} \\
\hline
\end{tabular}

NB: 0 is the standard deviation of $y$-intercepts of calibration curves, $\mathrm{S}$ is the slope of the calibration curve. 
Quantification of adenosine and cordycepin in cordyceps samples

The developed method was applied and successfully quantify amount of adenosine and cordycepin in natural $C$. sinensis and in cordyceps capsules. Chromatogram of $C$. sinensis was shown in Figure 4. The quantitative results of 28 samples were shown in Table 4. It was noticed that the amount of cordycepin in sample 2 was less than the LOQ and adenosine was not detected in sample 27. High variation level of adenosine and cordycepin among the samples was found in this study. The content of adenosine and cordycepin varied from not detected to $1.613 \mathrm{mg} / \mathrm{capsule}$ and not detected to $3.435 \mathrm{mg} /$ capsule, respectively.

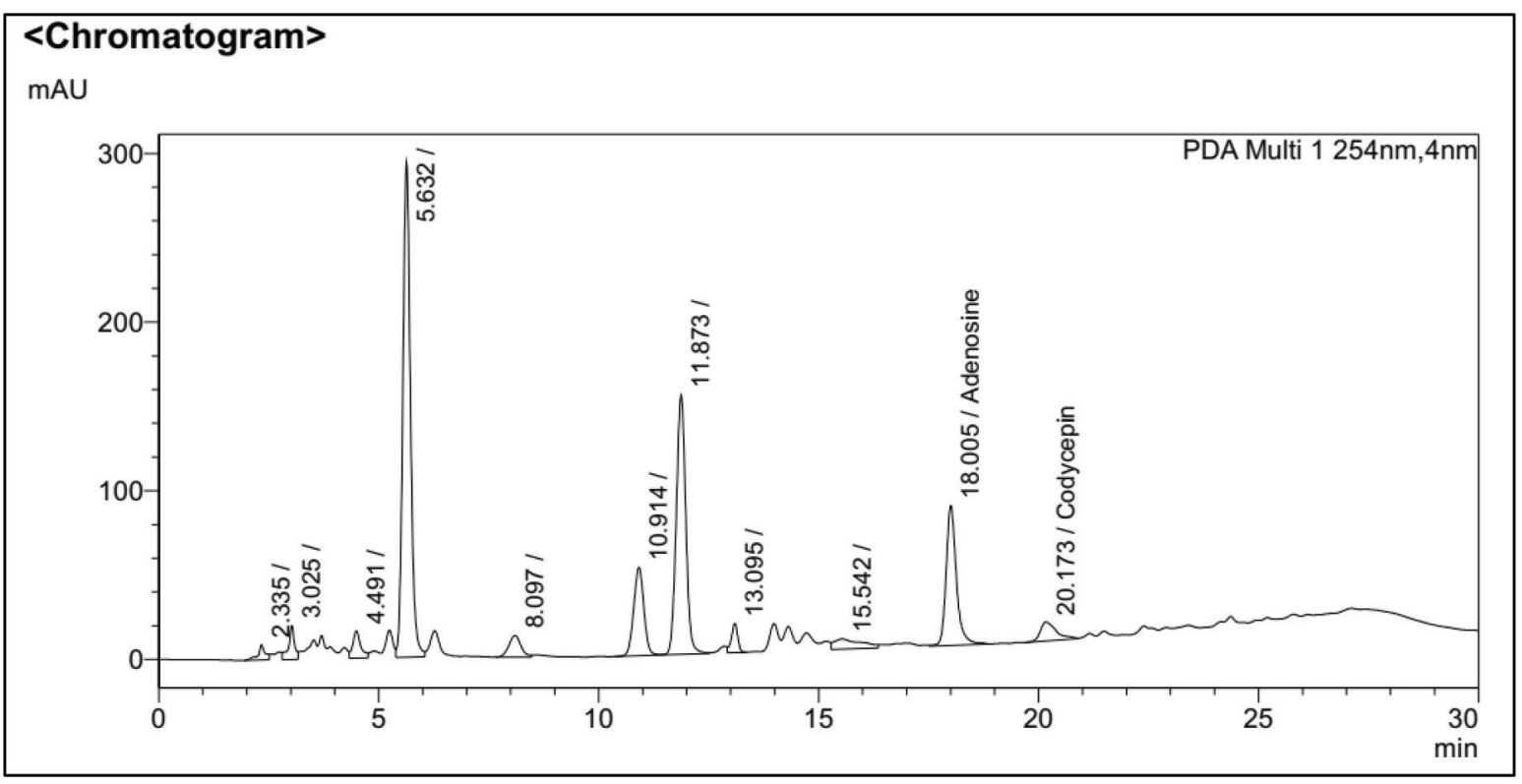

Figure 4. The chromatographic profile of natural C. sinensis obtained from the method in this study

Table 4. The amount of adenosine and cordycepin contained in one capsule of the individual cordycep products.

\begin{tabular}{|c|c|c|}
\hline Sample code & $\begin{array}{c}\text { Amount of adenosine } \\
\text { (mg per capsule) }\end{array}$ & $\begin{array}{c}\text { Amount of cordycepin } \\
\text { (mg per capsule) }\end{array}$ \\
\hline 1 & 0.063 & 0.052 \\
2 & 0.026 & ND* \\
3 & 0.106 & 1.051 \\
4 & 0.009 & 0.142 \\
5 & 0.172 & 0.066 \\
6 & 1.275 & 0.316 \\
7 & 1.169 & 0.234 \\
9 & 0.054 & 0.193 \\
10 & 0.269 & 1.434 \\
11 & $1.025^{* *}$ & 2.284 \\
12 & 1.364 & $2.210^{* * *}$ \\
13 & 0.084 & 0.121 \\
14 & 0.028 & 0.186 \\
15 & 0.074 & 0.227 \\
\hline
\end{tabular}


Table 4. The amount of adenosine and cordycepin contained in one capsule of the individual cordycep products.

\begin{tabular}{|c|c|c|}
\hline Sample code & $\begin{array}{c}\text { Amount of adenosine } \\
\text { (mg per capsule) }\end{array}$ & $\begin{array}{c}\text { Amount of cordycepin } \\
\text { (mg per capsule) }\end{array}$ \\
\hline 16 & 0.025 & 0.046 \\
18 & 0.421 & 1.085 \\
19 & 0.172 & 0.548 \\
20 & 0.169 & 0.895 \\
21 & 0.067 & 0.509 \\
22 & 0.263 & 0.437 \\
23 & 0.047 & 0.222 \\
26 & 0.053 & 0.107 \\
27 & $N D^{*}$ & 0.172 \\
28 & 0.156 & 0.480 \\
29 & 1.613 & 3.435 \\
31 & 0.437 & 0.898 \\
32 & 0.312 & 0.378 \\
34 & 0.168 & 0.275 \\
\hline
\end{tabular}

NB: ${ }^{*}$ Not detected (ND), ${ }^{* *}$ The peak purity index was $0.8235 .{ }^{* * *}$ The peak purity index was 0.8530 .

\section{DISCUSSION}

Most of the cordyceps products in this study were in formulation form; contained many variety of health promotors e.g. herbs (Ginseng, Lingzhi mushroom, Kaempferia parviflora, Angelica sinensis, Glycyrrhiza glabra, Coffee, Garlic, Turmeric), vitamins (Vitamin B1, Vitamin B2, Vitamin B6, Vitamin C, Choline, Coenzyme Q10), minerals (Zinc, Selenium), protein (L-Arginine, L-Glutathione, L-carnitine, Collagen, Oysters extract), polysaccharides (Yeast beta glucan, Inulin), etc. The quantitation of adenosine and cordycepin among interfering compounds in sample using HPLC-diode array detector developed in this study was more appropriate compare to other HPLCUV detector, reported previously ${ }^{2}$. The diode array detector is the key success of this method. It provides the peak purity index of the analytes which is the important tool for indicating the selectivity of the method. Although LC-MS/MS has better performance than HPLC-diode array detector. But LC-MS/MS is not the common instrument in laboratory nowadays. The limitation of LC-MS/MS has high cost either for the instrument or for the maintenance. Another advantage of this method was the sample preparation process. The method was intended to minimize and simplify the steps of sample preparation. After filtered through a $0.45 \mu \mathrm{m}$ Nylon syringe filter, the aqueous extract of the sample could directly inject into an HPLC system, there was no needed to dilute the filtrate. In addition, only one $15 \mathrm{~mL}$ test tube with screw cap glassware was needed for each sample preparation.

The criteria for quality control of fermented cordyceps capsules is very limited. The amount of active compounds, mannitol, adenosine and total amino acid contained in Bailing Jiaonang was controlled ${ }^{4}$. The limit of adenosine was not less than $0.16 \mathrm{mg}$ and $0.40 \mathrm{mg}$ for a 200 and a $500 \mathrm{mg}$ capsule, respectively. When the limit of adenosine in a $330 \mathrm{mg}$ capsule of Jinshuibao Jiaonang was $0.70-1.20 \mathrm{mg}$ and in Jinshuibao Pian, the limitation was controlled as the total of uridine, guanosine, and adenosine. There was no mention the use of cordycepin for quality control or as a marker of Cordyceps and related products. If this criterion for adenosine was applied to our study, only $50 \%$ or 14 out of 28 samples would pass the limit. However, the recommended dose of cordyceps 
capsule was in a wide range from 5 to 15 capsules, 3 times a day for a 8 weeks course treatment ${ }^{4}$. Anyhow, the Thai FDA should take action to control the quality of cordyceps products although cordyceps was considered to be safe ${ }^{6}$.

Cordyceps is known for numerous pharmacological effects, however, few adverse effects have been reported e.g. dry mouth, nausea, diarrhea and allergy. It is not recommended to use in patients who suffer from autoimmune diseases such as rheumatoid arthritis, systemic lupus erythematosus and multiple sclerosis. There is currently no available safety information on pregnant and lactating women ${ }^{6}$. The drug interaction of cordyceps may occur with the immunosuppressant such as azathioprine, basiliximab, cyclosporine, daclizumab, tacrolimus, sirolimus, prednisolone and corticosteroids.

\section{CONCLUSION}

The gradient HPLCdiode array detector method developed in this study is selective, precise, accurate and sensitive for separation and quantitation of adenosine and cordycepin in natural $C$. sinensis and in formulation of cordyceps capsules contained other health promotors.

\section{ACKNOWLEDGEMENTS}

The authors gratefully acknowledge the financial support provided by Thammasat University, Research Fund under the TU Research Scholar, contract No. 2/29/2560. We also would like to thank for the facilities support from the Faculty of Pharmacy, Thammasat University. We would like to thank Mrs. Yenjit Techadamrongsin for supplying natural Cordyceps sinensis. The appreciation is also for Dr.Nattapong Wichai and Dr.Chayanis Sutcharitchan for the information from Pharmacopoeia of the People's Republic of China.

\section{Conflict of interest (If any)}

There is no conflict of interest in this study.

\section{Funding}

None to declare

\section{Ethical approval}

None to declare

\section{Article info:}

Received September 6, 2019

Received in revised form February 24, 2020

Accepted May 30, 2020

\section{REFERENCES}

1. Techadamrongsin Y. Chongcao. J. Thai Trad. \& Altern. Med. 2014; 12(1): 3-16.

2. Li SP, Yang FQ, Tsim KW. Quality control of Cordyceps sinensis, a valued traditional Chinese medicine. J. Pharm. Biomed. Anal. 2006; 41(5):1571-84.

3. The Pharmacopoeia Commission of PRC (Eds.) Pharmacopoeia of the People's Republic of China, Beijing: Chemical Industry Publishing House. 2005.

4. Chinese Pharmacopeia Commission. Pharmacopoeia of People's Republic of China (Vol. I). Beijing: Chemical Medical Science Press. 2015. p. 832-833, 1072-5 (Chinese version).

5. Conti CR. Adenosine: Clinical pharmacology and application. Clin. Cardio. 1991; 14:91-3.

6. Tuli HS, Sandhu SS, Sharma AK. Pharmacological and therapeutic potential of Cordyceps with special reference to Cordycepin. Biotech. 2014;4: 1-12.

7. Yu L, Zhao J, Li SP, Fan H, Hong M, Wang YT, et al. Quality evaluation of Cordyceps through simultaneous determination of eleven nucleosides and bases by RP-HPLC. J.Sep. Sci. 2006;29: 953-8.

8. Van Iterson RA. Drenthe College Emmen Holland for www.standardbase.com. A Guide to Validation in HPLC based on the work of G.M. Hearn PERKIN ELMER. [document on the internet]. [cited 2019Aug 29]. Available from: http://www.parasshah.weebly.com> uploads $>$ hplc_validation_pe

9. The European Agency for the Evaluation of Medicinal Products, Human Medicines Evaluation Unit. ICH Topic Q2B, Validation of Analytical Procedures: Methodology. Step 4 Consensus guideline, 6 November 1996. 